\section{Stimulus relations in classical GSR conditioning}

\author{
WILLI H. BUTOLLO \\ University of Graz, A-8010 Graz, Austria
}

In an experiment for classical conditioning of GSR responses, Ss received CS-UCS pairs (light and 105-dB tones), which were scattered among context stimuli (tones) of either higher $(120 \mathrm{~dB})$ or lower $(95 \mathrm{~dB})$ intensity compared with that of the UCS. Systematically varied context stimuli were also presented to control groups (no CS-UCS pairing). The results show distinct contextual effects not only for UCR but also for CR. They favor an adaptation-level theory interpretation as opposed to a theory of sensitization. The effects of context stimuli on conditionability support a hypothesis based on relative arousal effects as opposed to the "global" arousal theory, which concerns speed of conditioning.

In order to develop a conditioned response (CR) in classical conditioning, two physically measurable stimuli (CS and UCS) are presented several times together. The stimulus characteristics are always measured in absolute values, and additional stimulus variations (context stimuli) are kept constant or ignored. L a t e ly, however, such "task-irrelevant" aspects have been systematically examined. Grings (1965), for instance, was concerned with the influence of verbalized awareness of the CS-UCS relation and cognitive activities in the conditioning procedures.

Two questions are investigated in this paper: (1) In what way does the unconditioned response (GSR) change when, in addition to the CS-UCS pairs (light-tone pairs), unconditioned context stimuli (UCCS) of the same modality, but of a higher or lower intensity than that of UCS, are presented? (As previous experiments in the psychology of perception show, these context effects can still be described with a psychophysical formula, but it includes parameters of the context stimuli as well as parameters of the standard stimuli.) (2) To what extent is the development of a conditioned response affected by the influence of the context on UCR?

In answer to the first question, two different predictions can be made. According to the adaptation-level theory, an adaptation to context stimuli UCCS is expected. The UCR will appear stronger or weaker, depending on the relative position of its UCS on the intensity continuum, compared with the position of the context stimuli UCCS.

Therefore, with very intensive UCCS and medium UCS, the

*This experiment was carried out during a British Council bursary at the Institute of Psychiatry in London. The author thanks Dr. Eysenck, Dr. Martin, and Mr. Law for their help. and thereby to an increased readiness prediction is that the UCR will become continuously weaker. In the case of soft UCCS, the UCR to the same medium UCS should become stronger. A theory of sensitization may give an alternative prediction. According to this theory, strong stimuli lead to an increased activation for reactions. If loud UCCS are scattered among stimuli with medium intensity, responses will appear stronger than when the UCCS is soft.

The effects on conditionability could be derived from a combination of each of the aforementioned theories with the arousal theory of conditionability (Eysenck, 1965). The latter says that with an increased general arousal, conditioning takes place more easily.

In order to examine these alternative predictions, the experiment described in this paper was carried out. Galvanic skin resistance (GSR) responses to tones (UCS) of medium loudness were chosen as UCR. There were two reasons for this choice:

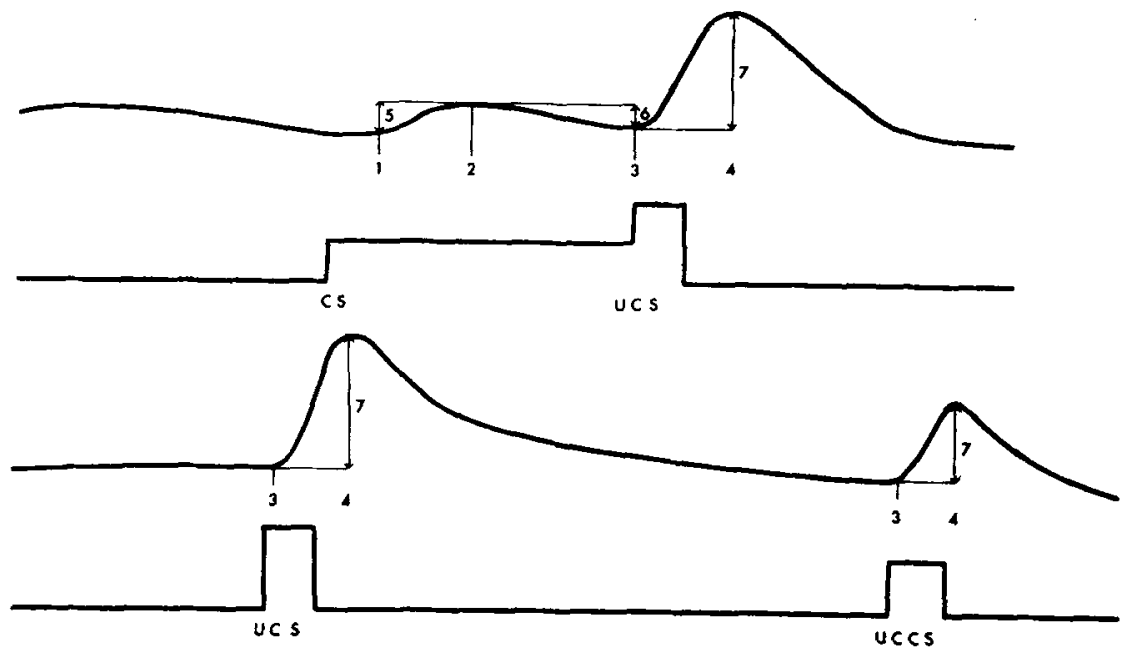

Fig. 1. GSR measures: (1) onset latency, (2) Peak I latency, (3) minimum latency, (4) Peak II latency, (5) Peak I amplitude, (6) minimum negative amplitude, (7) Peak II amplitude. (Ordinate: conductance.)
(1) GSR responses usually correlate positively with the intensity of the UCS (tone), by means of which relativity effects in perception should become well visible in the form of varying amplitudes. (2) Furthermore, the autonomous GSR response as an indicator of arousal is an important variable in psychological theories in general and especially in the theory and application of behavior therapy. So it seems to be very important to know the influence of stimulus context of a conditioning situation on acquisition and extinction of conditioned GSR responses.

\section{SUBJECTS}

Thirty-two Ss participated in this study (20 young housewives and 12 students of above 20 years of age). They received a reward for taking part in the experiment. Four Ss were assigned to each of the eight experimental groups.

In an orthogonal design, the independent variables were: (1) I-Intensity of context stimuli; two levels: louder (120 dB) vs softer (95 dB) than UCS (110 dB), i ndependent samples; (2) C-Conditioning: CS-UCS paired $\left(C_{1}\right)$ vs not paired $\left(C_{2}\right)$ during $C R$ acquisition (two levels), independent samples; (3) P-Context stimuli present $\left(P_{1}\right)$ or not present $\left(P_{2}\right)$ during the extinction period (two levels), i nde pendent samples; (4) T-Successive trials (25 context trials scattered among 10 conditioning trials), dependent samples. Dependent variables were amplitudes and measures of latency for response onset and peak.

GSR responses were recorded as described by Martin (1965): direct current device, $\mathrm{Ag} / \mathrm{AgCl}$ electrodes located on two fingers (palmar) of the 
Table 1

Cell Means of the Seven GSR Measures for $\mathrm{C}\left(\mathrm{C}_{1}, \mathrm{CS}-\mathrm{UCS}\right.$ Paired; $\mathrm{C}_{2}$, CS-UCS Not Paired), I ( $\left.I_{1}, U C C S=120 \mathrm{~dB} ; I_{2}, U C C S=95 \mathrm{~dB}\right)$, and $P\left(P_{1}\right.$, UCCS Present; $P_{2}$, UCCS Not Present)

\begin{tabular}{|c|c|c|c|c|c|c|c|c|c|}
\hline & & & \multicolumn{4}{|c|}{ Latencies (in Seconds) } & \multicolumn{3}{|c|}{$\begin{array}{l}\text { Response Amplitudes } \\
\text { (Logarithms of kOhms) }\end{array}$} \\
\hline & & & Onset & Peak I & Minimum & Peak II & Peak I & Minimum & Peak II \\
\hline \multicolumn{10}{|c|}{ CR Acquisition } \\
\hline & $C_{1}$ & $I_{1}$ & $\begin{array}{l}1.30 \\
1.25\end{array}$ & $\begin{array}{l}3.12 \\
3.25\end{array}$ & $\begin{array}{l}6.37 \\
6.14\end{array}$ & $\begin{array}{l}8.25 \\
8.63\end{array}$ & $\begin{array}{l}0.99 \\
1.26\end{array}$ & $\begin{array}{l}0.67 \\
0.96\end{array}$ & $\begin{array}{l}1.15 \\
1.96\end{array}$ \\
\hline & $\mathrm{C}_{2}$ & $\begin{array}{l}\mathrm{I}_{1} \\
\mathrm{I}_{2}\end{array}$ & $\begin{array}{l}1.49 \\
1.68\end{array}$ & $\begin{array}{l}3.49 \\
3.65\end{array}$ & $\begin{array}{l}8.02 \\
7.19\end{array}$ & $\begin{array}{r}10.05 \\
9.60\end{array}$ & $\begin{array}{l}1.25 \\
1.02\end{array}$ & $\begin{array}{l}0.29 \\
0.23\end{array}$ & $\begin{array}{l}0.31 \\
0.20\end{array}$ \\
\hline \multicolumn{10}{|c|}{ CR Extinction } \\
\hline \multirow{2}{*}{$P_{1}$} & $\mathrm{C}_{1}$ & $\begin{array}{l}I_{1} \\
I_{2}\end{array}$ & $\begin{array}{l}1.31 \\
1.74\end{array}$ & $\begin{array}{l}3.41 \\
3.93\end{array}$ & $\begin{array}{l}6.75 \\
6.07\end{array}$ & $\begin{array}{l}8.38 \\
7.58\end{array}$ & $\begin{array}{l}0.98 \\
0.47\end{array}$ & $\begin{array}{l}0.48 \\
0.08\end{array}$ & $\begin{array}{l}0.63 \\
0.26\end{array}$ \\
\hline & $\mathrm{C}_{2}$ & $I_{1}$ & $\begin{array}{l}1.08 \\
1.82\end{array}$ & $\begin{array}{l}2.42 \\
3.51\end{array}$ & $\begin{array}{l}5.30 \\
6.82\end{array}$ & $\begin{array}{l}6.87 \\
8.58\end{array}$ & $\begin{array}{l}0.73 \\
0.34\end{array}$ & $\begin{array}{l}0.24 \\
0.07\end{array}$ & $\begin{array}{l}0.45 \\
0.08\end{array}$ \\
\hline \multirow[b]{2}{*}{$\mathbf{P}_{2}$} & $c_{1}$ & $\begin{array}{l}I_{1} \\
I_{2}\end{array}$ & $\begin{array}{l}1.26 \\
0.94\end{array}$ & $\begin{array}{l}3.54 \\
2.96\end{array}$ & $\begin{array}{l}7.12 \\
6.79\end{array}$ & $\begin{array}{l}8.84 \\
8.90\end{array}$ & $\begin{array}{l}0.88 \\
1.36\end{array}$ & $\begin{array}{l}0.64 \\
0.92\end{array}$ & $\begin{array}{l}0.67 \\
1.25\end{array}$ \\
\hline & $\mathrm{C}_{2}$ & $I_{1}$ & $\begin{array}{l}1.43 \\
1.19\end{array}$ & $\begin{array}{l}3.34 \\
3.08\end{array}$ & $\begin{array}{l}6.56 \\
6.97\end{array}$ & $\begin{array}{l}8.84 \\
8.38\end{array}$ & $\begin{array}{l}0.57 \\
1.48\end{array}$ & $\begin{array}{l}0.31 \\
0.52 \\
\end{array}$ & $\begin{array}{l}0.35 \\
0.67 \\
\end{array}$ \\
\hline
\end{tabular}

Table 2

Cell Means for Measures of GSR Responses to Context Stimuli

\begin{tabular}{|c|c|c|c|}
\hline & $\begin{array}{l}\text { Onset Latency } \\
\text { (in Seconds) }\end{array}$ & $\begin{array}{l}\text { Peak I Latency } \\
\text { (in Seconds) }\end{array}$ & $\begin{array}{c}\text { Peak I Response } \\
\text { Amplitude (in } \\
\text { Logarithms } \\
\text { of kOhm) }\end{array}$ \\
\hline \multicolumn{4}{|c|}{ Context Stimuli (UCCS) } \\
\hline $\begin{array}{ll}\mathrm{C}_{1} & \mathrm{I}_{1}\end{array}$ & $\begin{array}{l}1.25 \\
1.19\end{array}$ & $\begin{array}{l}3.80 \\
2.65\end{array}$ & $\begin{array}{l}1.60 \\
0.78\end{array}$ \\
\hline $\begin{array}{ll}\mathrm{C}_{2} & \mathrm{I}_{1} \\
& \mathrm{I}_{2}\end{array}$ & $\begin{array}{l}0.93 \\
0.99\end{array}$ & $\begin{array}{l}3.03 \\
2.68\end{array}$ & $\begin{array}{l}1.58 \\
1.03\end{array}$ \\
\hline $\begin{array}{r}\text { UCR Control (W } \\
I_{1} \\
I_{2}\end{array}$ & $\begin{array}{l}1.32 \\
0.97\end{array}$ & $\begin{array}{l}3.24 \\
3.28\end{array}$ & $\begin{array}{l}1.10 \\
1.80\end{array}$ \\
\hline
\end{tabular}

left hand, bipolar recording system. Presentation of stimulus sequences was given by an especially programmed LINC-8 computer.

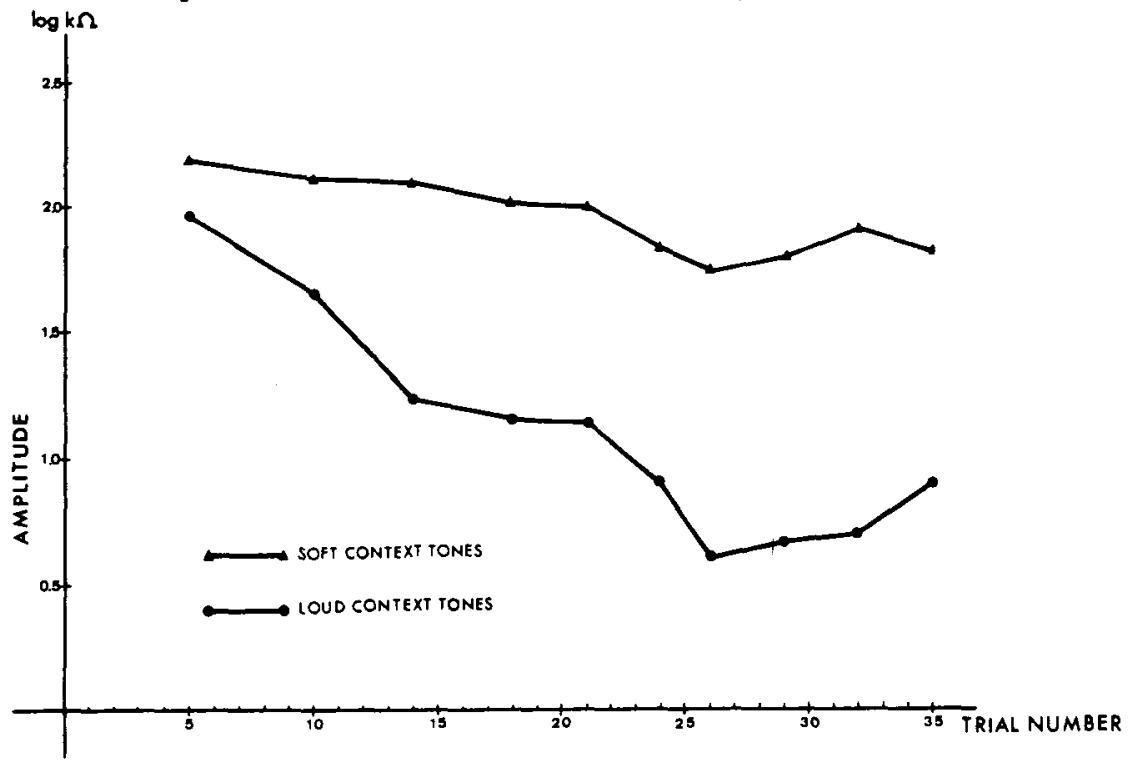

Fig. 2a. Effects of loud $\left(I_{1}, 120 \mathrm{~dB}\right)$ and soft $\left(I_{2}, 95 \mathrm{~dB}\right)$ context stimuli on amplitudes of GSR responses (UCR) to UCS of constant medium intensity (110 dB). Abscissa : 35 trials of 10 UCS and 25 UCCS mixed. for 1 sec. Both stimuli stopped at the same time. The time intervals between the CS-UCS pairs varied at random between 50 and 184 sec. During the acquisition period, 10 conditioning trials (CS-UCS paired) were presented after four standardization trials (one UCS, three CS alone). Together with the conditioning trials, another 25 UCCS were presented, scattered at random. In this series of 35 acoustic stimuli, two or three UCCS are therefore always followed by a CS-UCS pair. UCS-UCCS intervals and UCCS-CS/UCS intervals varied from 24 to 50 sec. The UCCS lasted 1 sec. Intensity of the "loud" context stimuli was $120 \mathrm{~dB}$, of the "soft" context stimuli, $95 \mathrm{~dB}$. As the control condition (Level $\mathrm{C}_{2}$ ) in Factor $\mathrm{C}$ (CS-UCS not paired), $10 \mathrm{CS}$ and 10 UCS were scattered at random among the UCCS.

During the extinction period, the CS was presented alone with approximately the same time intervals, either with UCCS $\left(P_{1}\right)$ or without $\operatorname{UCCS}\left(P_{2}\right)$ in between. All together there were another five single CS presentations.

\section{RESULTS}

The manually scored measures of the responses to $\mathrm{CS}-\mathrm{UCS}$ during acquisition and of responses to $\mathrm{CS}$ during extinction were: (1) latency of onset and peak of first response (CS) and its amplitude; (2) latency and negative amplitude of the minimum following the first peak (UCR onset); and (3) latency and amplitude of the second peak (UCS) (see Fig. 1). Data were submitted to univariate multifactorial analyses of variance and will now be discussed successively. The cell means of the seven measures are shown in Tables 1 and 2 for $C, I$, and $P$. The main results concerning changes over trials are shown in Figs. $2 a$ and 2b. [The scores for amplitudes have been logarithmically transformed (base 10). 1

Responses to Context Stimuli (Acquisition)

The intention of this analysis was to establish whether or not the different intensities of additional stimuli (UCCS), which are scattered among CS-UCS pairs, actually lead to different responses to UCCS. The logarithm of the amplitudes of responses shows significant differences at $p<.01$ between the intensity conditions, the responses to more intensive stimuli being stronger. The monotonous decrease of the amplitude of response with successive trials ( $T$ ) is significant at $\mathrm{p}<.02$. The interaction of $\mathrm{I}$ by $\mathrm{T}$ proved to be irrelevant. Evidence is thus furnished for a response habituation, which shows almost parallel curves for both degrees of intensity. 


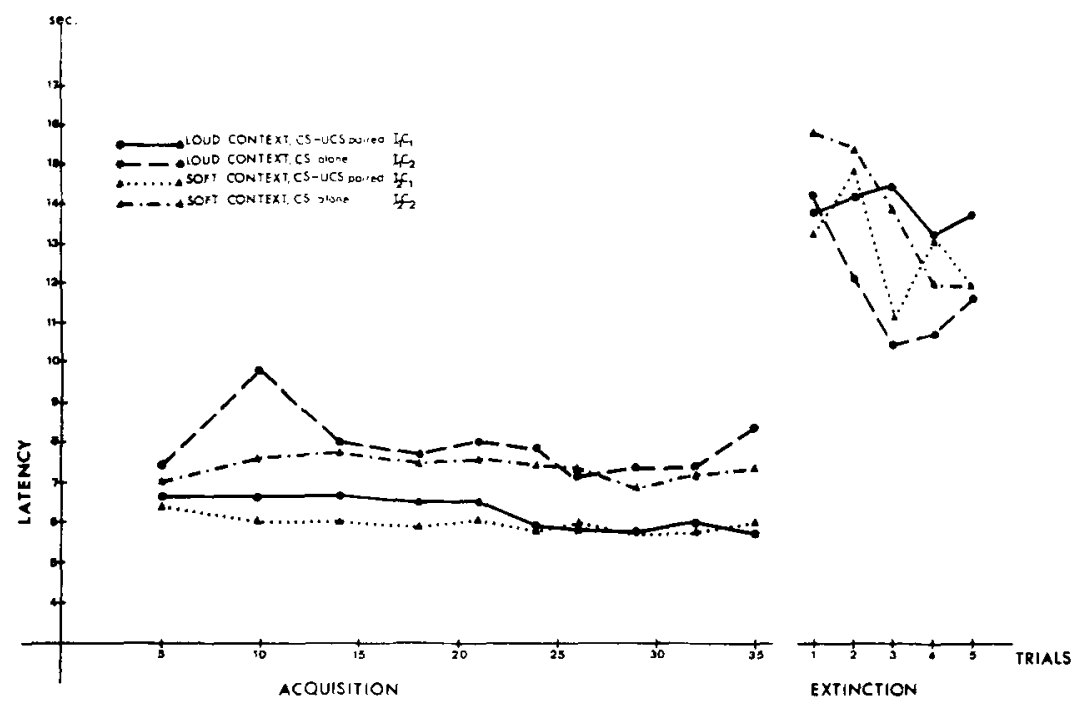

Fig. 2b. Effect of intensity of context stimuli (I) and conditioning on acquisition and extinction of $C R$ latency ("minimum latency"). $I_{1} C_{1}=$ loud context tones and CS-UCS pairing; $\mathrm{I}_{1} \mathrm{C}_{2}=$ loud context and no CS-UCS pairing; $I_{2} C_{1}=$ soft context tones and CS-UCS pairing; $I_{2} C_{2}=$ soft context tones and no CS-UCS pairing.

\section{Contextual Effects on}

UCR (Acquisition)

To show the effect of variations of UCCS intensity on characteristics of responses to physically constant UCS control data (no CS-UCS pairing) have been evaluated separately. The effect of intensity is, indeed, significant $(p<.03)$ : the responses to medium UCS among more intensive UCCS were distinctly weaker, yet stronger in the case of less intensive UCCS (see Fig. 2a).

Effects of UCCS on Conditioning of the Changed UCS (Acquisition)

It is expected that, above all, conditioning will affect the measure "latency" and "amplitude" of the minimum between both peaks (Scores 3 and 6), latency of the second peak (4), and amplitude of the first peak (5). In spite of physically constant UCS, the factor "intensity" (I) of UCCS leads to highly significant differences in amplitudes of the second peak $(\mathrm{p}<.001$; higher amplitude with softer UCCS) and in the typical measure of conditioning: "minimum latency" $\quad(p<.01$; intensive UCCS lead to longer latencies; see Fig. 2b). Factor $\mathrm{C}$ (conditioning vs control) is highly significant, not only for "minimum latency" $(p<.001)$, but also for "minimum amplitude" $(p<.01)$. Minimum latency: CS-UCS pairing leads to shorter minimum latencies and larger minimum amplitudes. Naturally, Factor $\mathrm{C}$ is also significant for the amplitude of the second peak $(p<.001)$, but this indicates only that the UCS presented after the CS causes a further strong response on the part of the Ss of the conditioning groups.
Such responses did not occur in the control group, where the CS was presented alone. The interaction of $\mathrm{I}$ by $\mathrm{C}$ is significant for the minimum latency at $\mathrm{p}<.02$. For the amplitude of the second peak I by C shows significance at the $\mathrm{p}<.001$ level. While the main effect "trials" seems to be irrelevant, the interaction of $\mathrm{T}$ by $\mathrm{C}$ is significant for the minimum latency at $\mathrm{p}<.05$.

\section{Contextual Effects}

During Extinction

Two kinds of contextual influences must be distinguished: (1) effects of context during acquisition, which are still effective during extinction, although UCCS are not presented; and (2) effects of UCCS presented during extinction. Factor $P$ (UCCS present or not) controls each of these possible influences.

It could not be proved that UCCS intensity affects one of the seven measures during extinction. Factor $\mathrm{C}$ is significant for the amplitudes of the second peak $(\mathrm{p}<.03 ;$ minimum amplitude, $\mathrm{p}<.12$ ).

The influence of Factor $P$ is relatively distinct for all three measures of amplitude. When UCCS are present, the amplitudes for the first and second peak are smaller. Also, the interaction of $\mathrm{P}$ by $\mathrm{I}$ for onset latency $(p<.02)$ and amplitudes of first $(p<.02)$ and second peak $(p<.02)$ show significance. When the UCCS is present and more intensive, the amplitudes of responses to $\mathrm{CS}$ extinction period, when the soft UCCS of the acquisition trials is no longer presented $\left(P_{2}\right)$. The main effect, $T$ (onset latency, latency of first peak, increases. It decreases during the minimum latency, latency and amplitude of second peak), the interactions of $\mathrm{T}$ by I (onset latency), $\mathrm{T}$ by $\mathrm{C}$ (latency of first peak), and $T$ by $P$ (onset latency), as well as the interactions of $\mathrm{T}$ by $\mathrm{P}$ by I (onset latency, latency of first peak) and $\mathrm{T}$ by $\mathrm{P}$ by $\mathrm{I}$ by $\mathrm{C}$ (onset latency, latency of the first peak) show very high significance $(p<.01)$. Conditionability

The significant interaction of $\mathrm{I}$ by $\mathrm{C}$ of minimum latency during $C R$ acquisition indicates that the contextual variation affects the conditionability $(p<.02)$. In order to put this effect under control, the differences between the two contextual conditions in the number of CS-UCS trials, which are necessary to reduce reliably the minimum latency, have been additionally tested (U test). The almost significant U values obtained show a probability of $p<.08$ for the null hypothesis, This, together with the significant interaction of $\mathrm{I}$ by $\mathrm{C}$, implies to a certain extent that soft UCCS cause a more rapid conditioning.

\section{DISCUSSION}

The results show a strong effect of the context stimuli, which favors the adaptation-level theory as opposed to a theory of sensitization. GSR responses to tones of medium intensity are smaller, when these tones are presented among a series of very intensive tones (UCCS) (contrast effect). Besides this, the fact that responses to UCCS themselves habituate parallelly in the two UCCS intensity conditions does not support an interpretation according to the sensitization theory either. Moreover, it became evident that the different responses to UCS, which are dependent on the stimulus context, were conditionable, i.e. context-dependent difference in UCR appear also in CR.

The amplitude of responses to CS (first peak) and especially the minimum latency, which measures the genesis of the conditioned response during acquisition trials, varied with the CS-UCS pairing condition according to UCCS, although the physical properties of UCS remained constant (conditioning itself was repeatedly proved).

\section{REFERENCES}

EYSENCK, H. J. Extraversion and the acquisition of eyeblink and GSR conditioning responses. Psychological Bulletin, 1965, 63, 258-270.

GRINGS, W. W. Verbal-perceptual factors in the conditioning of autonomous responses. In $W$, F. Prokasy (Ed.), Classical conditioning. New York: Appleton-Century-Crofts, 1965.

HELSON, H. Adaptation level theory. New York: Harper, 1964.

MARTIN, I. Discriminatory GSRs. Activitas Nervosa Superior, 1965, 7, 217-223. 